SHS Web of Conferences 21, 00001 (2015)

DOI: $10.1051 /$ shsconf/20152100001

C) Owned by the authors, published by EDP Sciences, 2015

\title{
Liste des auteurs
}

Bernard Marie-Claude, Professeure adjointe

Département d'études sur l'enseignement et l'apprentissage

Faculté des Sciences de l'éducation de l'Université Laval, Québec, QC

CRIRES - Centre de recherche et d'intervention sur la réussite scolaire

Membre de l'ASIHVIF - Association internationale des histoires de vie en formation

marie-claude.bernard@fse.ulaval.ca

Browaeys Dorothée, Journaliste

Coordinatrice volet français du programme européen Synenergene, coordinatrice du Forum NanoRESP, Rédactrice en chef adjointe d'UP'Magazine

dorbrow@gmail.com

Charles Frédéric, MC

Laboratoire S2HEP, EA 4148, Université Claude Bernard Lyon 1 et École Normale Supérieure de Lyon, France charles@univ-lyon1.fr

Chérif Nadia, Enseignante

Lycée Belkaid, Bir El Djir, Oran, Algérie

Coquide Maryline, Professeure didactique des sciences

STEF Sciences Techniques Éducation Formation ENS de Cachan

ENS Lyon - INRP

maryline.coquide@ens-lyon.fr 
De Montgolfier Sandrine, Maître de conférences, histoire des Sciences du Vivant IRIS - CNRS/INSERM/EHESS/P13

Université Paris Est Créteil Val de Marne (UPEC), ESPE de Créteil sandrine.demontgolfier@u-pec.fr

Dell'Angelo Michèle: Maître de conférences didactique des sciences / sciences de la vie et de la Terre

UMR STEF Sciences Techniques Éducation Formation ENS de Cachan

Université Paris Est Créteil (UPEC), France

michele@dellangelo.com

Dahmani Hassen Reda, Enseignant-chercheur

Département des Sciences Naturelles, ENS, Vieux-Kouba,Alger, Algérie dahmani@ens-kouba.dz

Dargent Géraldine, Enseignante de SVT

Centre International de Valbonne, Sophia Antipolis Valbonne olivier.dargent@free.fr

Dargent Olivier Enseignant de SVT

Centre International de Valbonne, Sophia Antipolis Valbonne olivier.dargent@free.fr

Fortin Corinne, Enseignant-chercheur didactique des sciences / sciences de la vie et de la Terre

STEF Sciences Techniques Éducation Formation ENS de Cachan

Université Paris Est Créteil (UPEC)

corinne.fortin@u-pec.fr

Grancher Céline, doctorante en didactique des sciences / ATER

E3D-LACES, Université de Bordeaux, ÉSPÉ d'Aquitaine, France celine.grancher@u-bordeaux.fr

Harvey Léon, Professeur

Doyen aux affaires départementales et étudiantes de l'UQAR

Université du Québec à Rimouski (UQAR)

leon_harvey@uqar.ca

Kramer Ijsbrand M. Enseignant chercheur

Université de Bordeaux, France

i.kramer@iecb.u-bordeaux.fr 
Lhoste Yann, Maître de conférences, HDR en sciences de l'éducation, didactique des SVT

E3D-LACES / Université de Bordeaux, ÉSPÉ d'Aquitaine, Bordeaux, France yann.lhoste@espe-aquitaine.fr

Molinatti Grégoire, Enseignant-chercheur

Faculté d'Éducation, LIRDEF Université de Montpellier et Centre Norbert

Elias, Marseille, France

gregoire.molinatti@univ-montp2.fr

Noack Julie, Doctorante en philosophie, anthropologie

ENS de Lyon et Université de Montréal

julie.noack@ens-lyon.fr

Parizeau Marie-Hélène, professeure titulaire

Faculté de Philosophie de l'Université Laval, Québec, QC

Vice-présidente de la Commission mondiale d'éthique des connaissances scientifiques et des technologies (COMEST) de l'UNESCO

Directrice du groupe de recherche en éthique médicale et environnementale marie-helene.parizeau@fp.ulaval.ca

Pautal Éliane, enseignant chercheur

UMR EFTS Toulouse 2 Jean Jaurès et ESPE Académie de Limoges, France eliane.pautal@unilim.fr

Piron Florence, professeure titulaire

Département d'information et de communication de l'Université Laval, Québec, QC

Présidente de l'Association Science et bien commun

Dirige le projet Science ouverte en Haïti et Afrique francophone (SOHA)

florence.piron@com.ulaval.ca

\section{Roby Catherine, ATER}

CREAD, Université Rennes 2, France

catherine.roby@orange.fr

Samson Ghislain, Professeur titulaire

Département des sciences de l'éducation de l'Université du Québec à Trois-

Rivières (UQTR)

Ghislain.Samson@UQTR.ca

Schneeberger Patricia, Professeur en sciences de l'éducation, didactique des SVT E3D-LACES, Université de Bordeaux, ÉSPÉ d'Aquitaine, France patricia.schneeberger@u-bordeaux.fr 
Simard Catherine, Professeure invitée

Faculté des sciences de l'éducation à l'Université du Québec à Rimouski (UQAR)

Didactique des sciences et des technologies

Catherine_Simard@uqar.ca

Simonneaux Laurence, Enseignante-chercheure

ENFA Castanet Tolosan, France

laurence.simonneaux@educagri.fr

Triquet Eric, Professeur, Sciences de l'information et de la communication Université d'Avignon, France eric.triquet@univ-avignon.fr

Vidal Michel, Formateur-chercheur

Montpellier Sup Agro, Florac, France

michel.vida199@educagri.fr 